\title{
Magnetic Resonance Imaging of Bacterial Meningoencephalitis in a Foal
}

\author{
Judit Viu, ${ }^{1}$ Lara Armengou, ${ }^{1}$ Cristian de la Fuente, ${ }^{2}$ Carla Cesarini, ${ }^{1}$ \\ Sònia Añor, ${ }^{2}$ and Eduard Jose-Cunilleras ${ }^{1}$ \\ ${ }^{1}$ Servei de Medicina Interna Equina, Departament de Medicina i Cirugia Animals, Facultat de Veterinària, \\ Universitat Autònoma de Barcelona, Bellaterra, 08193 Barcelona, Spain \\ ${ }^{2}$ Servei de Neurologia i Neurocirurgia, Departament de Medicina i Cirugia Animals, Facultat de Veterinària, \\ Universitat Autònoma de Barcelona, Bellaterra, 08193 Barcelona, Spain
}

Correspondence should be addressed to Lara Armengou, lara.armengou@uab.es

Received 23 May 2012; Accepted 16 August 2012

Academic Editors: L. Arroyo and C. Cantile

Copyright ( $\odot 2012$ Judit Viu et al. This is an open access article distributed under the Creative Commons Attribution License, which permits unrestricted use, distribution, and reproduction in any medium, provided the original work is properly cited.

\begin{abstract}
Magnetic resonance imaging (MRI) in equidae suffering meningoencephalitis (ME) has not been described. The objective of this paper is to describe brain MRI findings in a foal with bacterial ME. A five-month-old, $200 \mathrm{~kg}$ bwt Arabian filly was referred with a history of abnormal mental status and locomotion. The filly was recumbent and obtunded, and pupillary light reflexes were sluggish, and oculocephalic movements were normally present. Ophthalmic examination revealed bilateral optic neuritis. Hematology revealed leukocytosis and neutrophilia. Cerebrospinal fluid analysis showed neutrophilic pleocytosis with intracellular bacteria. On brain MRI, there were multifocal cortical areas of mild hyperintensity on T2-weighted images (T2WI) affecting both hemispheres. The lesions had ill-delineated margins, and there was loss of differentiation between gray and white matter. Diffuse hyperintensity was also identified in the left cerebellar cortex on T2WI. Neither mass effect nor cerebral midline shift were identified. On FLAIR images, the lesions were also hyperintense and, in some areas, they seemed to coalescence to form diffuse cortical areas of hyperintensity. The MRI findings described were similar to the MRI features described in cases of humans and small animals with ME. Brain MRI can be a useful diagnostic tool in foals and small-sized equidae with intracranial disease.
\end{abstract}

\section{Introduction}

Magnetic resonance imaging (MRI) is a very useful imaging technique in human neurology since the eighties. The use of MRI in veterinary medicine is very common in small animals, specially in those with neurological disease, but the use in horses is less frequent. The most common application of MRI in equine medicine is in the diagnosis of muscular or skeletal problems. The use of MRI in equine neurology is restricted due to limitations of size and weight-bearing capacity of most MRI units. However, due to the smaller size and lower weight of foals, MRI has been used in these animals and the findings reported for several cases of neonatal equine neurological disorders. Nevertheless, to the authors' knowledge, there are no reports describing the MRI lesions of bacterial meningoencephalitis (ME) in foals. This paper describes these lesions and demonstrates how MRI can be extremely useful in the diagnosis of ME in foals.

\section{Case Details}

A five-month-old, $200 \mathrm{~kg}$ bwt Arabian filly was referred to the Unitat Equina of the Hospital Clinic Veterinari de la UAB for evaluation of abnormal mental status and generalized profound weakness. The filly had began to show signs of progressive lethargy a week prior to admission, that progressed to right thoracic limb paresis and inability to stand at 12 hours before referral. According to the referring veterinarian, the filly was born at term but it showed confusion and inability to nurse. Five mares and their foals as well as the filly's dam lived together and none of them showed 
any neurological or physical alterations. All animals were in regular vaccination and deworming programs.

Physical examination on admission revealed that the filly was recumbent and markedly obtunded. Its body condition and hair coat were normal. No swollen joints or wounds were observed. Mild tachycardia $(60 \mathrm{bpm})$ and tachypnea $(48 \mathrm{bpm})$ were detected, and gastrointestinal sounds were decreased. Body temperature was within normal limits $\left(37.6^{\circ} \mathrm{C}\right)$, and no cervical stiffness or painful areas were detected on palpation. On neurological examination, pupillary reflexes were sluggish bilaterally, and oculocephalic movements were normally present. No other alterations were detected in cranial nerve or spinal reflex evaluation. Propioception tests could not be performed due to recumbency. The results of the neurological examination were consistent with an intracranial lesion affecting diffusely the cerebral hemispheres. Ophthalmological examination revealed papillary congestion with mild peripapillar exudates in both eyes and uveitis with small amounts of fibrin in the anterior chamber of the left eye.

Radiographs of the cervical vertebrae and skull were apparently normal. Thoracic radiographs revealed an alveolar pattern consistent with subclinical pneumonia. Hematology and plasma biochemistry performed upon admission revealed mildly increased blood lactate concentration $(3.2 \mathrm{mmol} / \mathrm{L}$, reference value $<2 \mathrm{mmol} / \mathrm{L})$, [1] marked leukocytosis $(20,110$ cells $/ \mu \mathrm{L}$, reference value 5,400 $14,300$ cells $/ \mu \mathrm{L})[1]$ and neutrophilia $(16,210$ cells $/ \mu \mathrm{L}$, reference value $2,200-8,600$ cells $/ \mu \mathrm{L}$ ) [1], and normal values of packed cell volume, total plasma protein, and fibrinogen concentrations. Plasma sodium, potassium, and chloride concentrations were within normal limits, and no alterations were detected in acid-base status. Increased creatine kinase (CK 6782 IU/L, reference range 60-266 IU/L) [1] and aspartate transaminase (AST 512 IU/L reference range 280$520 \mathrm{IU} / \mathrm{L})$ [1] activities were also detected.

Cerebrospinal fluid (CSF) was obtained under general anesthesia from the atlanto-occipital space, and an MRI study of the brain was performed under the same anesthesia with a $0.2 \mathrm{~T}$ open permanent magnet (VetMR; Esaote, Genoa, Italy). The foal was premedicated with romifidine $(0.03 \mathrm{mg} / \mathrm{kg} \mathrm{IV})$ and butorphanol $(0.03 \mathrm{mg} / \mathrm{kg} \mathrm{IV})$. The anaesthesia was induced with diazepam $(0.05 \mathrm{mg} / \mathrm{kg}$ IV) and thiobarbital $(4 \mathrm{mg} / \mathrm{kg}$ IV) and maintained in lateral recumbency with isoflurane in 100\% oxygen. Total anesthetic time was 2 hours. For MRI, the foal was kept in left lateral recumbency with the neck extended and the head against a linear coil. Transverse spin-echo T1-weighted images (T1WI; TR $=590 \mathrm{~ms}, \mathrm{TE}=26 \mathrm{~ms}$, slice thickness $=6 \mathrm{~mm}$, interslice gap $=0.6 \mathrm{~mm}$ ), transverse and sagittal T2-weighted images $(\mathrm{T} 2 \mathrm{WI}$; TR $=3090 \mathrm{~ms}$, TE $=80 \mathrm{~ms}$, slice thickness $=6 \mathrm{~mm}$, interslice gap $=0.6 \mathrm{~mm})$, and transverse FLAIR images $(\mathrm{TR}=7860 \mathrm{~ms}, \mathrm{TE}=90 \mathrm{~ms}, \mathrm{TI}=1500 \mathrm{~ms}$, slice thickness $=6 \mathrm{~mm}$, interslice gap $=0.6 \mathrm{~mm}$ ) were obtained. In addition, transverse T1WI were acquired after intravenous (iv) injection of gadopentate dimeglumine (Magnevist, Bayer Schering Pharma, Berlin, Germany.) (0.1 mmol/ $\mathrm{kg})$. The mean obtention time for each sequence was 15 minutes, and the total scanning time was one hour and a half. MR

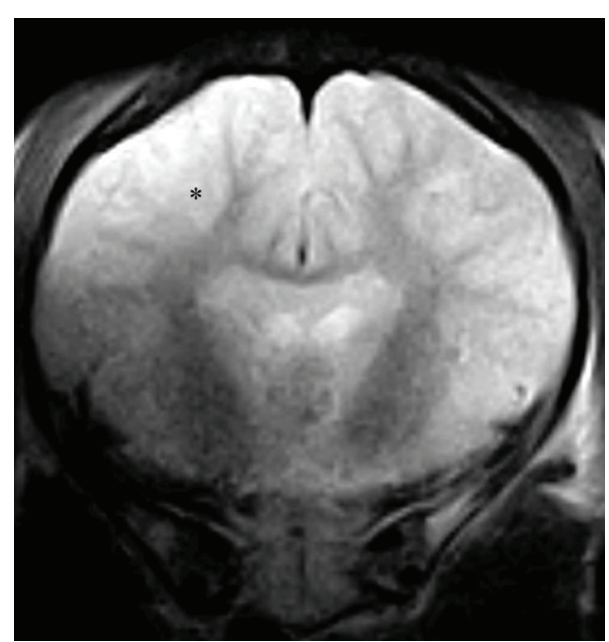

Figure 1: Transverse T2-weighted image at the level of rostral diencephalon showing a cortical area of mild hyperintensity with loss of differentiation between gray and white matter in the right hemisphere $(*)$.

images were loaded and evaluated with a Medical Imaging Software (OsiriX, open source software; http://www.osirixviewer.com, Macintosh platform). MRI showed multifocal cortical areas of mild hyperintensity on T2WI affecting both hemispheres. The lesions had diffuse margins, and there was loss of differentiation between gray and white matter. Although both hemispheres were affected, the lesions were more severe in the cortical gray matter of the frontal and parietal lobes of the right hemisphere (Figure 1). Diffuse hyperintensity also was identified affecting the left cerebellar cortex on T2WI. Neither mass effect nor cerebral midline shift was identified. On precontrast T1WI, the lesions were isointense to normal gray matter (Figure 2). On FLAIR images the lesions were hyperintense and, in some areas, they tended to coalescence to form diffuse cortical areas of hyperintensity. Some cortical areas that had normal appearance in T1WI and T2WI showed high signal intensity on FLAIR images (Figure 3). These changes were more evident in the parietal and temporal lobes of the right cerebral hemisphere, where diffuse FLAIR hyperintensity was detected. Due to cerebrospinal fluid signal suppression, areas of mild periventricular hyperintensity were more easily identified on FLAIR images than on T2WI. On postcontrast T1WI, only mild gyral enhancement was seen in both frontal lobes. Most lesions did not enhance after contrast administration. Ther was no meningeal enhancement either. The MR imaging findings were considered consistent with either an infectious-inflammatory process (encephalitis), or a multifocal vascular (ischemic) condition affecting the cerebral and cerebellar cortices.

Analysis of CSF revealed normal total protein concentration $(76.5 \mathrm{mg} / \mathrm{dL}$, reference range $10-120 \mathrm{mg} / \mathrm{dL})[1]$ and neutrophilic pleocytosis $(15$ cells $/ \mu \mathrm{L}$, reference range $0-7$ cells $/ \mu \mathrm{L}$ ) [1], with intracytoplasmic bacillus identified on cytology. Blood and CSF cultures were not performed 


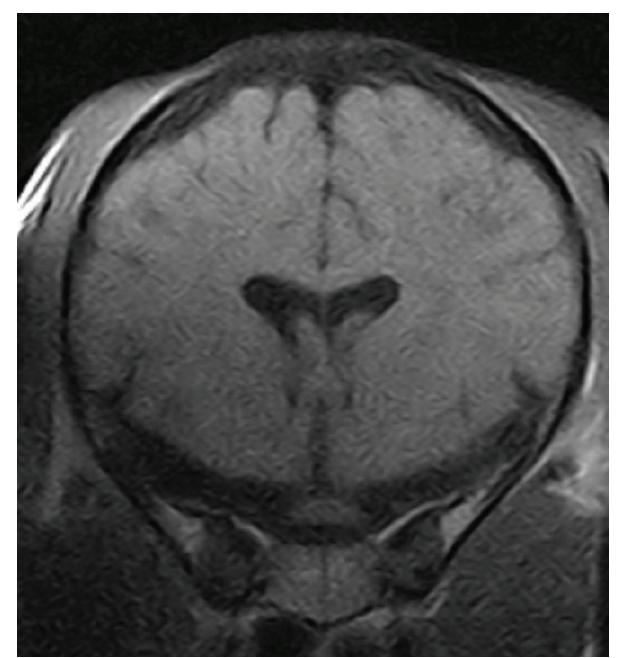

Figure 2: Transverse T1-weighted image at the level of rostral diencephalon showing isointensity of the lesion identified on T2weighted and FLAIR images.

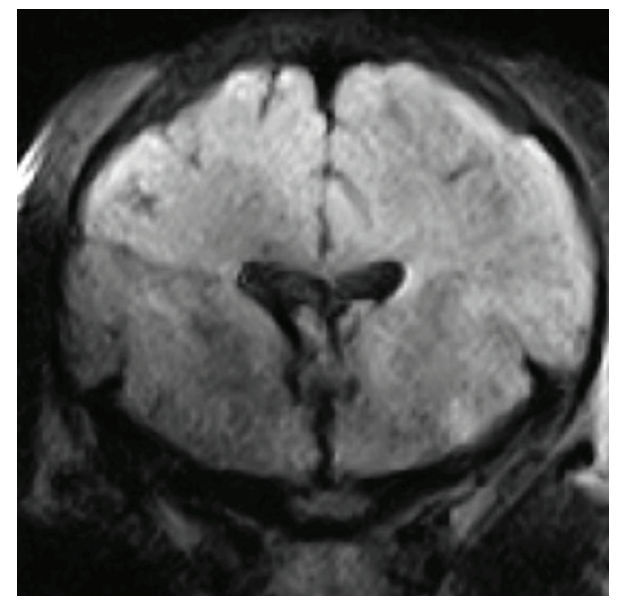

FIgURE 3: Transverse FLAIR image at the level of rostral diencephalon showing multiple hyperintense areas in the cerebral cortex of both hemispheres.

because of lack of availability of a microbiology laboratory at that time.

Based on history, clinicopathological findings, and diagnostic test results, a presumptive diagnosis of ME including bilateral optic neuritis was given, with associated diagnosis of left eye uveitis, pneumonia, and mild myositis.

The foal received iv and oral antimicrobials (sulfadiazinetrimethoprim and rifampin, resp.). Intravenous sodiumrich crystalloid (Lactated Ringer's plus $10 \mathrm{~mL} / \mathrm{L}$ of $7.5 \%$ $\mathrm{NaCl}$ ) and iv colloidal solutions (6\% hydroxyethyl starch) were administered according to the animal requirements. Intranasal oxygen supplementation, iv $2 \%$ dimethyl sulfoxide solution, and iv flunixin meglumine were also administered. Topical ocular therapy consisting of broad spectrum antibiotic and nonsteroidal anti-inflammatory drugs (NSAIDs) was applied. Once the foal recovered from general anesthesia, it began to show hyperexcitability, intention tremors, and vestibular signs. After 12 hours of treatment, the filly's mental status improved and at 24 hours after treatment initiation, it was able to stand up for short periods of time. Paresis of the right thoracic limb was consistently observed when the filly was standing.

Radiographs of the right thoracic limb showed no evident alterations. After a week of treatment, hematology and plasma muscle enzyme activities normalized, but the animal still showed paresis of the right thoracic limb upon discharge from the hospital. Systemic antimicrobial and NSAID treatments were continued for two weeks after leaving the hospital.

Oral communication with the referring veterinarian confirmed full recovery by the time medical therapy was interrupted without persistence of neurological or orthopedic signs.

\section{Discussion}

Bacterial meningitis is an uncommon condition in mature horses. In adult horses, the condition has been related to infectious diseases involving the head and $[2,3]$ trauma or common variable immunodeficiency [4]. In young horses, Streptococcus equi equi meningoencephalomyelitis [5], S. equi equi abscessation $[2,6]$, and intracranial Rhodococcus equi abscessation [7] have been described. ME has been identified as a complication in $8-10 \%$ of septicemic foals $[8,9]$. Cytological CSF findings in foals with meningitis usually include neutrophilic pleocytosis and increased protein concentration; however, normal or moderately increased values have also been reported in few cases. [10] The CSF obtained in the foal herein reported had a normal protein concentration with a mild neutrophilic pleocytosis and evidence of intracellular bacteria on cytology. The mild alterations found on CSF analysis were probably due to the predominance of encephalitis over meningitis, and perhaps the presence of nonexudative lesions found mainly in the brain parenchyma. Normal CSF analysis cannot completely rule out inflammatory central nervous system (CNS) disease in veterinary cases [11]. Because of this, MRI is highly recommended in cases of suspected inflammatory brain disease to improve the diagnostic yield $[12,13]$.

The MRI study was performed using a low-magnetic field unit $(0.2 \mathrm{~T})$ designed for small animal imaging. Because of this, positioning of our patient and coil selection were limited. In addition, low field MRI is time consuming if highresolution images, small slice thickness, and large regions, of study are desired. In order to minimize anesthetic time, temporal resolution (minimum acquisition time to achieve diagnostic images prevailing over high resolution images) was elected. So large slice thickness/interslice gap and small image matrixes were selected to improve signal-noise ratio and reduce acquisition time. Lesion morphology, topography, and magnetic resonance signal characteristics observed in this case were similar to the MRI features described in humans and small animals with meningoencephalitis, although these patterns are not pathognomonic $[14,15]$. In 
the case reported herein, the lesions were mildly hyperintense on T2WI but they were better visualized and delineated in the FLAIR images. FLAIR sequences have been advocated as a very useful MRI technique to detect inflammatory brain lesions due its high sensitivity over T2WI [16, 17]. In addition, periventricular white matter involvement is well depicted due to CSF signal suppression on FLAIR images. These features were also observed in this case and allowed better assessment of lesion extension. Paramagnetic contrast enhancement was mild and only observed in lesions affecting both frontal lobes, showing a gyral pattern. This contrast enhancement pattern has also been described in human infectious ME but it is not disease-specific [14]. Although meningeal contrast uptake is not always present in confirmed cases of meningitis in other species [15], the absence of meningeal, either dural or leptomeningeal, contrast enhancement strongly suggested brain parenchyma involvement rather than meningeal involvement.

Cerebellar lesions were also detected on the MRI study. However, the filly presented mild vestibulocerebellar signs only for a few hours in the postanesthetic period. The lack of clinical evidence of vestibulocerebellar signs on initial presentation could be explained by a predominance of diffuse bilateral hemispheric signs causing a profoundly obtunded mental status and hiding other concomitant neurolocalizations. Another possible explanation is a drug-related exacerbation of vestibulocerebellar signs in the postanesthetic period. Although there are not many studies in the literature about the effect of sedatives or anesthetic agents on the neurologic status of patients with CNS disorders, it has been postulated that some anesthetic agents could induce worsening of neurologic signs, specially vestibulocerebellar signs [18].

Bacterial meningitis is a disease difficult to diagnose and usually carries a poor prognosis. To the authors' knowledge, there are no previous descriptions of the MRI findings in horses with bacterial ME. The similarity of the herein reported findings to those observed in bacterial ME cases in other species leads to consider MRI as a very helpful diagnostic tool in foals.

\section{References}

[1] K. T. T. Corley and J. Stephen, "Appendix," in The Equine Hospital Manual, T. T. Corley and J. Stephen, Eds., pp. 654689, Blackwell, 1st edition, 2008.

[2] J. J. Smith, P. J. Provost, and M. R. Paradis, "Bacterial meningitis and brain abscesses secondary to infectious disease processes involving the head in horses: seven cases (19802001)," Journal of the American Veterinary Medical Association, vol. 224, no. 5, pp. 739-742, 2004.

[3] E. Mitchell, M. O. Furr, and H. C. McKenzie, "Bacterial meningitis in five mature horses," Equine Veterinary Education, vol. 18, no. 5, pp. 249-255, 2006.

[4] A. Pellegrini-Masini, A. I. Bentz, I. C. Johns et al., "Common variable immunodeficiency in three horses with presumptive bacterial meningitis," Journal of the American Veterinary Medical Association, vol. 227, no. 1, pp. 114-122, 2005.

[5] C. Finno, N. Pusterla, M. Aleman et al., "Streptococcus equi meningoencephalomyelitis in a foal," Journal of the American
Veterinary Medical Association, vol. 229, no. 5, pp. 721-724, 2006.

[6] A. Pellegrini-Masini and L. C. Livesey, "Meningitis and Encephalomyelitis in Horses," Veterinary Clinics of North America, vol. 22, no. 2, pp. 553-589, 2006.

[7] J. C. Janicek, J. Kramer, J. R. Coates, J. C. Lattimer, A. M. LaCarrubba, and N. T. Messer, "Intracranial abscess caused by Rhodococcus equi infection in a foal," Journal of the American Veterinary Medical Association, vol. 228, no. 2, pp. 251-253, 2006.

[8] B. D. Brewer and A. M. Koterba, "Bacterial isolates and susceptibility patterns in foals in a neonatal intensive care unit," Compendium on Continuing Education for the Practicing Veterinarian, vol. 12, pp. 1773-1781, 1990.

[9] A. M. Koterba, B. D. Brewer, and F. A. Tarplee, "Clinical and clinicopathological characteristics of the septicaemic neonatal foal: review of 38 cases," Equine Veterinary Journal, vol. 16, no. 4, pp. 376-382, 1984.

[10] M. O. Furr, "Bacterial infections of the central nervous system," in Equine Neurology, M. O. Furr and S. Reed, Eds., pp. 187-194, Wiley-Blackwell, 1st edition, 2008.

[11] K. G. Braund, Clinical Neurology in Small Animals: Localization, Diagnosis and Treatmen, International Veterinary Information Service Ithaca, Ithaca, NY, USA, 2002.

[12] A. A. Bohn, T. B. Wills, C. L. West, R. L. Tucker, and R. S. Bagley, "Cerebrospinal fluid analysis and magnetic resonance imaging in the diagnosis of neurologic disease in dogs: a retrospective study," Veterinary Clinical Pathology, vol. 35, no. 3, pp. 315-320, 2006.

[13] A. Splendiani, E. Puglielli, R. De Amicis, S. Necozione, C. Masciocchi, and M. Gallucci, "Contrast-enhanced FLAIR in the early diagnosis of infectious meningitis," Neuroradiology, vol. 47, no. 8, pp. 591-598, 2005.

[14] O. Kastrup, I. Wanke, and M. Maschke, "Neuroimaging of infections," NeuroRx, vol. 2, no. 2, pp. 324-332, 2005.

[15] S. Hecht and W. H. Adams, "MRI of brain disease in veterinary patients part 2: acquired brain disorders," Veterinary Clinics of North America-Small Animal Practice, vol. 40, no. 1, pp. 3963, 2010.

[16] K. Tsuchiya, S. Inaoka, Y. Mizutani, and J. Hachiya, "Fast fluidattenuated inversion-recovery MR of intracranial infections," American Journal of Neuroradiology, vol. 18, no. 5, pp. 909913, 1997.

[17] G. B. Cherubini, S. R. Platt, S. Howson, E. Baines, D. C. Brodbelt, and R. Dennis, "Comparison of magnetic resonance imaging sequences in dogs with multi-focal intracranial disease: PAPER," Journal of Small Animal Practice, vol. 49, no. 12, pp. 634-640, 2008.

[18] G. D. Thal, M. D. Szabo, M. Lopez-Bresnahan, and G. Crosby, "Exacerbation or unmasking of focal neurologic deficits by sedatives," Anesthesiology, vol. 85, no. 1, pp. 21-25, 1996. 

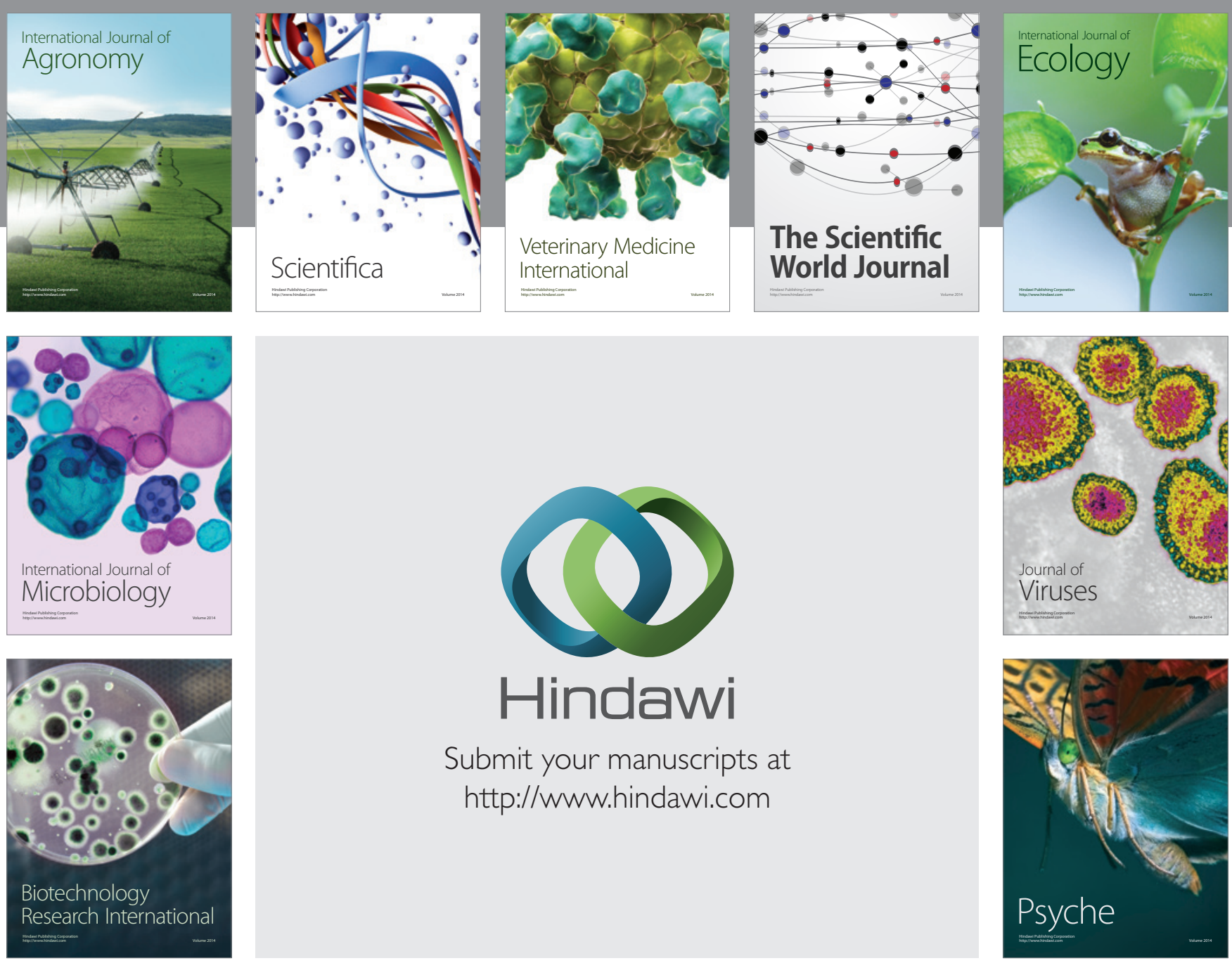

Submit your manuscripts at

http://www.hindawi.com
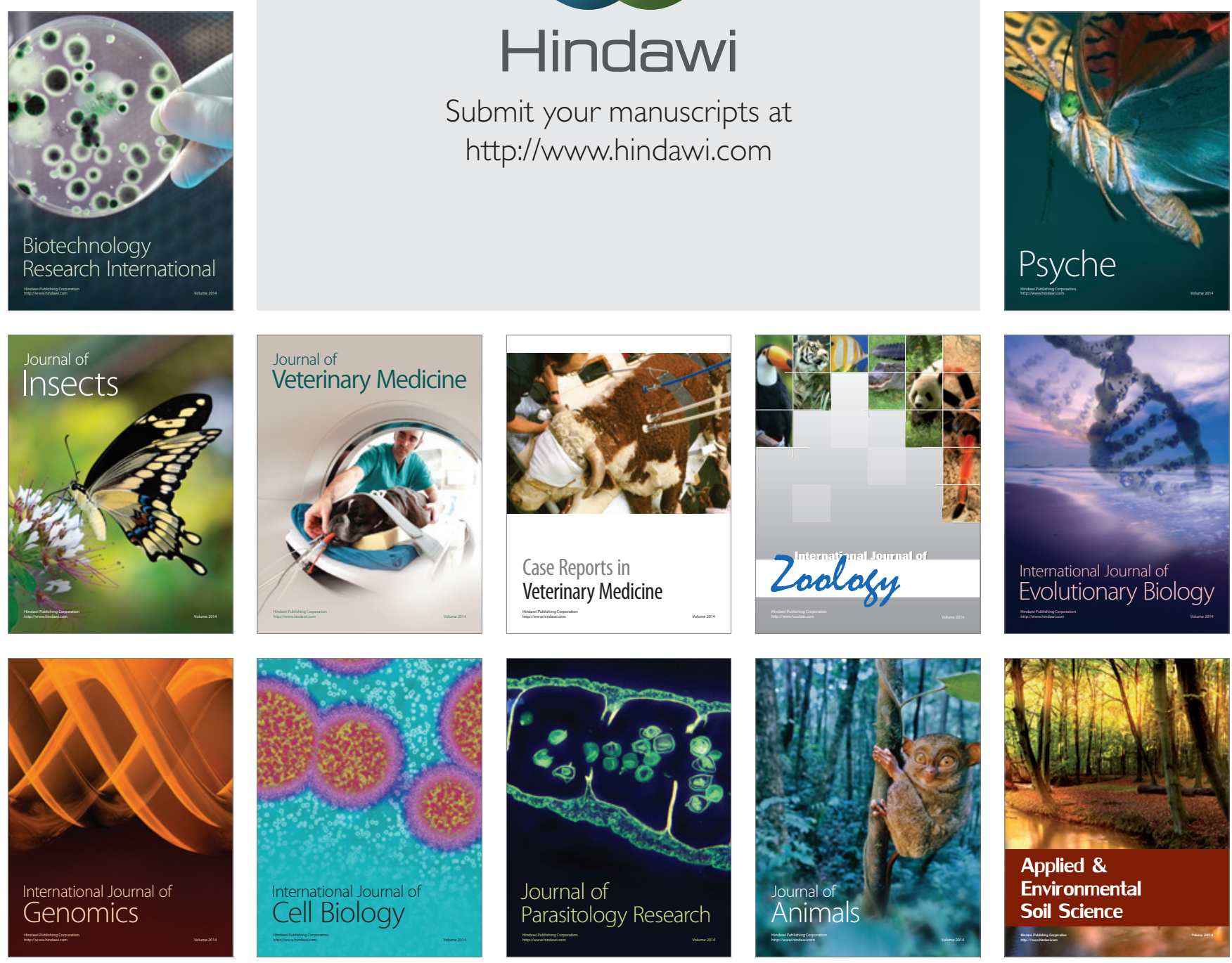